\title{
Carotid artery stenosis and inflammatory biomarkers: the role of inflammation-induced immunological responses affecting the vascular systems
}

\author{
Tissa Wijeratne ${ }^{1,2,3}$, Rohit Menon ${ }^{4}$, Carmela Sales ${ }^{4}$, Leila Karimi $^{5,6}$, Sheila Crewther $^{5} \wedge$ \\ ${ }^{1}$ Department of Neurology, AIMSS, WHCRE level three, Sunshine Hospital and Melbourne Medical School, St Albans, Victoria, Australia; ${ }^{2}$ School \\ of Public health and Psychology, La Trobe University, Bundoora, Victoria, Australia; ${ }^{3}$ Department of Medicine, Faculty of Medicine, Rajarata \\ University, Saliyapura, Anuradhapura, Sri Lanka; ${ }^{4}$ Department of Neurology and Stroke Service, Western Health, AIMSS, level 3, WHCRE, \\ Sunshine Hospital, St Albans, Victoria, Australia; ${ }^{5}$ School of Psychology and Public Health, La Trobe University, Melbourne, Australia; ${ }^{6}$ Ivane \\ Javakhishvili Tbilisi State University, Tbilisi, Georgia \\ Contributions: (I) Conception and design: T Wijeratne; (II) Administrative support: T Wijeratne; (III) Provision of study materials or patients: All \\ authors; (IV) Collection and assembly of data: All authors; (V) Data analysis and interpretation: All authors; (VI) Manuscript writing: All authors; (VII) \\ Final approval of manuscript: All authors. \\ Correspondence to: Tissa Wijeratne. Professor \& Chair, Department of Neurology, AIMSS, WHCRE level three, Sunshine Hospital and Melbourne \\ Medical School, St Albans, 3021, Victoria, Australia. Email: twi@unimelb.edu.au.
}

\begin{abstract}
The death, disability and economic cost of stroke are enormous. Indeed, among the 16 million people worldwide who suffer a stroke' annually, nearly six million die, and another five million are left permanently disabled making prevention of stroke one of the most important priorities in healthcare. Currently carotid artery stenosis (CS) or narrowing of the common carotid artery (CCA) or internal carotid artery (ICA) due to atherosclerotic plaque, accounts for $20-30 \%$ of all ischemic strokes. Atherosclerosis is now regarded as a chronic inflammatory disease in response to vascular compromise especially from hypertension. This has long been known to lead to inflammation and atherosclerotic plaque formation in the blood vessels. This mini-review aims to highlight the role of inflammation and neuro-immunological processes in carotid artery disease. Various cellular elements of inflammation and advanced imaging techniques have been identified as potential markers of plaque progression. Therapies related to decreasing and modulating immune-responsive inflammation in the carotid vessels have been shown to translate into decreased occurrence of acute neurologic events and improvement of clinical outcomes.
\end{abstract}

Keywords: Stroke; Carotid artery stenosis (CS); atherosclerosis; hypertension; stroke prevention; biomarkers; inflammation

Submitted May 31, 2020. Accepted for publication Jul 10, 2020.

doi: $10.21037 /$ atm-20-4388

View this article at: http://dx.doi.org/10.21037/atm-20-4388

\section{Introduction}

Stroke is the second leading cause of mortality and a leading cause of adult disability in the world (1-7). The majority of strokes are of the ischaemic subtype, with $20-40 \%$ suffering from intracranial atherosclerotic disease (8). An estimate of
$20-30 \%$ of all strokes are attributed to extracranial carotid artery stenosis (CS), most frequently occurring at the bifurcation of the internal and external carotid artery (9). The prevalence of CS in individuals over 80 years of age is approximately $5-7 \%$ in women and $9-12 \%$ in men, with

^ ORCID: Tissa Wijeratne, 0000-0002-1701-7111; Sheila Crewther, 0000-0002-1315-7563. 
$1 \%$ and $3 \%$ respectively having high-grade CS (defined as $70 \%$ stenosis or reduction of diameter of $>70 \%$ ) $(10,11)$. Hypertension is an important risk factor for the development of atherosclerosis and inflammation (12). Other risk factors for atherosclerosis includes unhealthy blood cholesterol levels, diabetes, overweight/obesity, unhealthy diet (food that are high in trans-fat and saturated fat, cholesterol and sodium) ,smoking, insulin resistance, older age (men the risk increases after age of 45 years, women risk increases after the age of 55 years), family history of lipid disorders, sleep apnoea, stress and agerelated inflammation is emerging as a specific risk factor for atherosclerosis.

\section{Inflammation and plaque atherogenesis: the role of acute and chronic inflammatory processes}

Atherosclerosis is central to the pathogenesis of CS. Hansson et al. describes this process as a long-standing, maladaptive disease which is anchored on the principles of the Virchow's triad: (I) stasis, (II) hypercoagulability, and (III) endothelial dysfunction (13). The simple view of atherosclerosis as a disorder of abnormal lipid deposition is redefined by complex, chronic, infllammatory process. This maladaptive, non resolving process occurs at sites of blood flow disturbance. Subendothelial retention of cholesterol containing lipoproteins and flow mediated inflammatory changes within the endothelial cells trigger the process of atherogenesis (13). Monocyte derived macrophages, $T$ cells interspersed with lipids and debris from dead cells can be found in these lesions. Cells of these lesions demonstrate on going features of inflammation with $\mathrm{T}$ cells, macrophages, proinflammatory cytokines with pro and anti-inflammatory signals $(5,6,13)$. Jonasson et al. noted the important interactions between smooth muscle cells and blood born cells in the pathogenesis of atherosclerosis (14). Additionally, soluble Vascular Cell Adhesion Molecule (sVCAM-1), and interleukin-6 (IL-6) have also been demonstrated to be present ubiquitously whenever high cholesterol-laden plasma lipoproteins are present in blood $(15,16)$. Interestingly, pathologic studies of carotid plaques also validated the presence of Chlamydia pneumoniae which has been associated with increased levels of high sensitive C-reactive protein (hs-CRP) and IL-6, both linked to acute coronary and vascular events (17). Various authors have proposed that the presence of this organism incites plaque progression (presence of Chlamydia in blood will have the same effect) in coronary and carotid specimens which results in prolonged arterial wall infection $(17,18)$. Despite an ongoing inflammation present in its core, the plaque is made stable largely due to the presence of an overlying intact endothelium which may remain for years without any overt clinical manifestations highlighting the potential time for treatment of high lipid levels and clinical signs of compromise (19).

Various morphological features differentiate symptomatic from asymptomatic CS. Histopathological evaluation of endarterectomy specimens show that plaque rupture, fibrous cap thinning, and fibrous cap foam-cell infiltration was strikingly more prominent (20). Biomarkers such as monocyte chemotactic protein, which is essential in the transmigration of monocytes and macrophages across the endothelium has been shown to be higher in patients with symptomatic CS $(21,22)$. Compared to their asymptomatic counterparts, patients with symptomatic CS also have higher white blood cell and neutrophil count, and neutrophil to lymphocyte ratio (NLR) (23). NLR represents the rise in the level of neutrophil, a marker of any type of acute inflammation coupled with the decrease in the lymphocyte count which is more related to persistent physiological stress (24) or low-level chronic inflammation (25). The same trend was observed by Varim et al. with regards to platelet to lymphocyte ratio among patients with severe CS. This increase is likely attributed to megakaryocytic proliferation mediated by the inflammatory mediators, IL-1 and IL-6 (26). Another marker which has been shown to be increased in patients with symptomatic CS is the Soluble Urokinase Plasminogen Activator Receptor (suPAR) which is ubiquitous in various cells involved in atherogenesis such as monocytes, neutrophils, activated T-lymphocytes, macrophages, endothelial cells, and smooth muscle cells (27). Circulating suPAR levels are elevated in various medical conditions associated with inflammation such as kidney disease, increasing age, diabetes, atherosclerosis, heart failure, sepsis, human immunodeficiency virus, autoimmune diseases, and smoking $(28,29)$.

\section{Inflammatory markers associated with carotid stenosis}

The presence of atherosclerotic lesions in carotid arteries has been linked with hypertension and increased serum levels of inflammatory markers leading to induced oxidative stress in the walls of the vascular system (12). The cells of atherosclerotic plaque are a secondary source of cytokines which have both local and systemic actions causing further inflammatory processes. This leads to the 
development of plaques that protrude into the arterial lumen as they progress, causing arterial stenoses and eventually occlusions (30). Debing et al. 2008, compared 180 gender age-matched controls and 180 patients with stenosis of the internal carotid artery (ICA), and noted higher levels of hsCRP, (sVCAM, IL-6 in the carotid endarterectomy (CEA) group (30) and proposed that these inflammatory markers could serve as biomarkers for CS. The best validated of these markers (for CS) are hsCRP (16,31,32), IL-6 and (33). Osteopontin (OPN), an integrin-binding ligand has a role in atherosclerosis as it increases the inflammation in the atherosclerotic plaque (34). Patients with CS ( $n=40)$ showed a two fold increase in OPN levels with positive correlations with intraplaque count of total neutrophils, macrophages and MMP-9 content in both symptomatic $(n=40)$ and asymptomatic $(\mathrm{n}=185)$ patients $(35)$. OPN promises to be a clinical as well as therapeutic tool for atherosclerosis and carotid stenosis with immense research interest for the future. Carotid intima-medial thickness (c-IMT), (thickness of the carotid arteries), has also been used as a surrogate marker for the extent of carotid atherosclerosis by Ammirati and colleagues who associated greater progression of cIMT with high levels of inflammatory markers (10). In addition, Puz et al. demonstrated a link between increased inflammatory markers and destabilisation of plaques (32). While fluctuations in the level of these biomarkers may indicate changes in the level of stenosis and likelihood of having an unstable plaque, a causal relationship between inflammatory biomarkers and the actual existing degree of carotid stenosis is yet to be established $(10,32,36)$. Rather it is highly likely that any type of inflammation induces higher levels of cytokines and if persistent will induce permanent vascular compromise (37).

Other examples of potential biomarkers for CS include serum amyloid A (SAA), lipoprotein-associated phospholipase A2 (Lp-PLA2), matrix metallo-proteinases (MMP), IL-1B, IL-10 and sVCAM, adiponectin, CD40 ligand, Leptin, infectious agents such as Chlamydia pneumonia, Helicobacter pylori, cytomegalovirus seropositivity $(16,38)$.

\section{Comparison of the effects of carotid endarterectomy (CEA) and carotid artery stenting (CAS) surgery}

Inflammatory biomarkers have been shown to be useful in carotid revascularization procedures. By looking at the serum S100 protein levels, which is a marker of cerebral injury, it has been demonstrated patients with vulnerable plaques have higher blood levels of the former and was more pronounced in patients undergoing CAS (39). A prospective cohort including 225 patients with severe CS undergoing CAS also showed that elevated TNF levels correlated with the occurrence of new DWI lesions post-surgery (40). The higher serum TNF- $\alpha$ levels noted to be associated with higher likelihood of new DWI lesions after CAS and the presence of intraplaque haemorrhage (IPH) (40).

Zuniga and colleagues also showed that inflammatory biomarkers such as IL-12p40, SDF1- $\alpha$ and TNF correlated with the occurrence of cognitive dysfunction after CEA or CAS (41). When compared with carotid stenting (55 patients), endarterectomy (43 patients) demonstrated postoperative improvement in cognition at one and six months compared with the baseline. Carotid stenting and age older than 80 years were associated with significant long-term cognitive impairment. Moreover, multiple inflammatory cytokines such as IL-12p, Stromal cellderived factor 1 alpha (SDF-1 $\alpha$ ), TNF- $\alpha$ was associated with a higher risk of significant cognitive impairment at one month. SDF- $1 \alpha$, TNF- $\alpha$ were independent predictors of cognitive impairment while IL- 6 showed cognitive protective effects at six months after the revascularization procedure (41).

\section{Inflammatory processes in symptomatic and asymptomatic CS}

\section{Imaging biomarkers of inflammation}

Apart from systemic biomarkers, molecular imaging has been shown to correlate significantly with carotid plaque inflammation. Fundamental to this is the use of PET with F-fluoro-2-deoxy-D-glucose and 18F-Fluorocholine $\left({ }^{18} \mathrm{~F}-\mathrm{FDG}\right)$ which reflect the degree of macrophage infiltration (42-44). Patients with symptomatic CAS have been shown to have greater uptake or "hot spots", particularly in macrophage-rich areas of the plaque which also translates to greater plaque vulnerability (43). Magnetic resonance imaging (MRI) has also been employed in the identification of inflammation by using gadolinium to track kinetic changes with respect to phagocyte activation and mobilization (45). In the ATHEROMA trial, ultrasmall superparamagnetic iron oxide (USPIO)-enhanced carotid MRI has been shown to correlate significantly with carotid plaque inflammation (46). BIOVASC (Biomarkers/Imaging Vulnerable Atherosclerosis in Symptomatic Carotid disease), a multicentre cohort study ( $\mathrm{n}=109,14$ recurrent strokes) showed 
that plaque inflammation related to ${ }^{18} \mathrm{~F}$-FDG uptake was able to predict future recurrent stroke post-PET (47).

Detection of active inflammation within a plaque is a major feature of a high-risk vulnerable plaque and vulnerable patient $(48,49)$.

\section{Vulnerable plaque and acute inflammatory processes resulting in plaque rupture}

Prior to the occurrence of a vascular event affecting the eye or the brain, various inflammatory processes come into play in a carotid atheroma making it susceptible to ulceration and subsequent rupture (50). Hence, it is important to identify features of plaque vulnerability in patients with symptomatic and asymptomatic CS to identify patient groups who are likely to benefit from urgent treatment. An unstable plaque is pathologically characterized by the presence of a thin fibrous cap, a large lipid core, the presence of intraplaque hemorrhage and the infiltration of various inflammatory cells such as monocyte-derived macrophages and activated smooth muscle cells $(13,51)$. Carr and colleagues have demonstrated that macrophages and T-lymphocytes expressing HLA-DR were predominant in endarterectomy specimens with evidence of plaque rupture (20). Proteolytic enzymes such MMPs and cathepsin cysteine proteases (CCP's), along with the depletion of their inhibitors promotes the process of plaque ulceration and subsequent rupture $(45,52)$. There is mounting evidence that the proteolytic enzyme MMP-9, a substance released by activated macrophages and capable of degrading the extracellular matrix, is more abundant in vulnerable plaques $(52,53)$. In addition, biomarkers which have been shown to be elevated in patients with high risk plaques include sCD40L, hs-CRP, TIMP-1, and TIMP-2 (53,54). The concept of angiogenesis is also essential to understanding of the patho-mechanisms of plaque vulnerability as it plays a role in enhancing vascular leakage and permeability $(55,56)$. Research has shown that vascular endothelial growth factor (VEGF) plays a dual role of being an angiogenic and inflammatory cytokine in patients with highly susceptible plaques (56).

Multimodal imaging of features such as the degree of inflammation, haemorrhage, neovascularity and the richness of the necrotic core by MRI has also been found to be useful in assessing the vulnerability of plaques (57). Techniques such as PET/CT have the advantage of being sensitive to the assessment of degree of inflammation in vulnerable plaques but are outweighed by MRI for their relative inability to assess anatomy, haemorrhage, and ulceration $(51,57)$. The degree of echolucency in carotid ultrasound is also related to elevated levels of macrophages and lipid which also translates to plaque vulnerability (58).

\section{Therapeutic potentials}

Over the years, the annual risk of stroke and transient ischemic stroke associated with carotid stenosis has decreased. Presumably because there have been major strides in scientific understanding of the pathogenesis of atherosclerosis and underlying inflammation which can lead to carotid stenosis. Such knowledge has led to both increased clinician awareness of biological risk factors and earlier diagnosis and more rigorous management and to public health awareness of diet and exercise routines (59). The management of carotid stenosis includes reduction of risk factors with lifestyle modification, pharmacological and/or surgical management, which may include CEA or CAS (60-63) and development of new therapeutic tools (64). In practice, pharmacological therapy should be instituted and optimised in all patients with carotid stenosis of higher risk requiring additional management beyond lifestyle modification. Modern intensive medical therapy is much safer than operative management, and surgical management is usually reserved for a subset of high-risk patients (61-63). A focus on a number of agents with anti-inflammatory properties has been reviewed (65).

Lipid-lowering therapy such as statins are extensively used in the prevention of atherosclerosis. In addition to its lipid-lowering mechanism of action, statins have antiinflammatory and antioxidant properties effective in reducing cardiovascular risk as demonstrated in numerous randomised double-blind controlled trials (66-68). Research validates that they prevent the increasing thickness of the carotid arteries (c-IMT) which has been used as a marker of the extent of atherosclerosis $(58,64,69)$ although this has many unfortunate side effects on cell membrane integrity in muscle and presumably elsewhere particularly brain where $>60 \%$ of weight is lipoprotein in cell membranes. It is noteworthy to remember the fact that healthy lipids such as omega-3s and omega-6s, are vital for overall brain and general health. Healthy lipids are important to stabilize the neuronal cell's structure in the brain. It can also reduce the inflammation and helps the immune system to function properly (70).

Research dictates that aspirin, an extensively used antiplatelet agent, has anti-inflammatory properties 
conferring a benefit in atherosclerosis management in addition to its other mechanisms of action $(66,67,71,72)$. Huo and colleagues demonstrated an association between folic acid (vitamin B9) and a reduction in both homocysteine levels (73) and the risk of stroke in a randomised trial involving 20,000 individuals (71). Herbs including turmeric, ginger and garlic and natural plants such as green tea have been postulated to have anti-inflammatory actions which may be beneficial in the prevention of atherosclerosis, however there have been no definitive clinical trials demonstrating this yet $(74,75)$.

Other drugs with anti-cytokine mechanisms typically used in other conditions have also been investigated. Colchicine is known to have anti-inflammatory actions and inhibits neutrophil function (76-79). It is widely used in the management of other inflammatory diseases such as gout and Behcet's disease which fuelled the rationale to investigate the potential role of colchicine in improving cardiovascular outcomes. The LoDoCo trial demonstrated colchicine prevented recurrent cardiovascular events in patients with stable coronary artery disease in addition to high dose statins (76-78). A drawback to colchicine is its narrow therapeutic index and many participants reported numerous intolerances. The COLCOT trial involving 4,745 individuals, concluded that low-dose colchicine significantly reduced ischaemic cardiovascular events $(77,78)$. Moving forward, the LoDoCo2 trial aims to further validate the role of colchicine and results of this have yet to be published $(77,79)$.

Methotrexate widely used in management of rheumatoid arthritis, has an anti-inflammatory action against TNF, IL-6, and CRP. The CIRT trial aimed to investigate-the role of methotrexate and its action against these (TNF, IL-r, and CRP) inflammatory markers and in resulting cardiovascular outcomes (79). There was however no reduction in cardiovascular events and inflammatory markers IL-1b, IL-6 or CRP with low-dose methotrexate compared to placebo (79).

Interest in the potential role of monoclonal human antibodies in preventing atherosclerosis has been the basis of a few clinical trials. Tocilizumab, a human monoclonal antibody against IL-6 receptor and etanercept, a TNF receptor antagonist, studied in ENTRACTE trial did not demonstrate a significant difference in cardiovascular outcomes in rheumatoid arthritis patients (80). The CANTOS trial involving 10,061 individuals compared canakinumab, an antibody against IL-1B with placebo. Findings showed that 3 -monthly canakinumab led to significantly lower rates of cardiovascular events compared to placebo (81).
The findings of upcoming clinical trials currently underway will provide more insights into the relationship between inflammation and atherosclerosis. It is promising that anti-inflammatory therapy may be beneficial in reducing atherosclerotic risk though, the consequences of interfering with immunity and host defence mechanisms across the whole body and with relation to the brain are still not fully understood. Careful consideration is required as these anti-inflammatory agents may potentiate the risk of infections. Whilst lifestyle modification remains a fundamental part of management, the availability of new pharmacological approaches enables us to expand our array of treatment strategies. The identification of sensitive and specific biomarkers for atherosclerosis will also further guide individualised therapy and achieve the goal of precision medicine.

\section{Conclusions}

The evidence reviewed here supports the concept of chronic inflammation as a contributory factor in atherosclerosis. Several studies have noted that better understanding of the biological control of biomarkers of inflammation are likely to predict potential impact of future vascular events. C-reactive protein measured as well as hsCRP, IL-6, IL-1 are among the front line of such potential biomarker lists.

Greater awareness of NLR (82-85) and PLR (85-90) have the potential to play more active roles in diagnosis and prognosis in the setting of symptomatic CS and are well worth exploring further in clinical and translational research.

Lastly better understanding of the therapeutic potential of immunomodulation in CS is an even more exciting treatment strategy. The promising results of the CANTOS trial are raising hopes of better therapeutic management of CS, i.e., potential for better management of one of the leading causes of death and disability worldwide.

\section{Acknowledgments}

Funding: None.

\section{Footnote}

Provenance and Peer Review: This article was commissioned by the Guest Editor (Dr. Kosmas I. Paraskevas) for the series "Carotid Artery Stenosis and Stroke: Prevention and Treatment Part I" published in Annals of Translational 
Medicine. The article was sent for external peer review organized by the Guest Editor and the editorial office.

Conflicts of Interest: All authors have completed the ICMJE uniform disclosure form (available at http://dx.doi. org/10.21037/atm-20-4388). The series "Carotid Artery Stenosis and Stroke: Prevention and Treatment Part I" was commissioned by the editorial office without any funding or sponsorship. The authors have no other conflicts of interest to declare.

Ethical Statement: The authors are accountable for all aspects of the work in ensuring that questions related to the accuracy or integrity of any part of the work are appropriately investigated and resolved.

Open Access Statement: This is an Open Access article distributed in accordance with the Creative Commons Attribution-NonCommercial-NoDerivs 4.0 International License (CC BY-NC-ND 4.0), which permits the noncommercial replication and distribution of the article with the strict proviso that no changes or edits are made and the original work is properly cited (including links to both the formal publication through the relevant DOI and the license). See: https://creativecommons.org/licenses/by-nc-nd/4.0/.

\section{References}

1. Feigin VL, Nguyen G, Cercy K, et al. The GBD 2016 Lifetime Risk of Stroke Collaborators. Global, Regional, and Country-Specific Lifetime Risks of Stroke, 1990 and 2016. N Engl J Med 2018;379:2429-37.

2. Feigin VL, Mensah GA, Norrving B, et al. Atlas of the Global Burden of Stroke (1990-2013): The GBD 2013 Study. Neuroepidemiology 2015;45:230-6.

3. Vosoughi K, Stovner LJ, Steiner TJ, et al. The burden of headache disorders in the Eastern Mediterranean Region, 1990-2016: findings from the Global Burden of Disease study 2016. J Headache Pain 2019;20:40.

4. Krishnamurthi RV, Feigin VL, Forouzanfar MH, et al. Global and regional burden of first-ever ischaemic and haemorrhagic stroke during 1990-2010: findings from the Global Burden of Disease Study 2010. Lancet Glob Health 2013;1:e259-81.

5. Feigin VL, Forouzanfar MH, Krishnamurthi R, et al. Global and regional burden of stroke during 1990-2010: findings from the Global Burden of Disease Study 2010. Lancet 2014;38:245-54.
6. Krishnamurthi RV, Moran AE, Forouzanfar MH, et al. The global burden of hemorrhagic stroke: a summary of findings from the GBD 2010 study. Glob Heart 2014;9:101-6.

7. Bennett DA, Krishnamurthi RV, Barker-Collo S, et al. The global burden of ischemic stroke: findings of the GBD 2010 study. Glob Heart 2014;9:107-12.

8. Banerjee C, Chimowitz MI. Stroke Caused by Atherosclerosis of the Major Intracranial Arteries. Circ Res 2017;120:502-13.

9. Prasad K. Pathophysiology and Medical Treatment of Carotid Artery Stenosis. Int J Angiol 2015;24:158-72.

10. Ammirati E, Moroni F, Norata GD, et al. Markers of inflammation associated with plaque progression and instability in patients with carotid atherosclerosis. Mediators Inflamm 2015;2015:718329.

11. Prasad M, Lerman LO, Lerman A. Carotid stiffness and cerebrovascular disease: the physiology beyond the anatomy. J Am Coll Cardiol 2015;66:2126-8.

12. Alexander RW. Hypertension and the Pathogenesis of Atherosclerosis. Hypertension 1995;25:155-61.

13. Hansson GK, Libby P, Tabas I. Inflammation and plaque vulnerability. J Intern Med 2015;278:483-93.

14. Jonasson L, Holm J, Skalli O, et al. Regional accumulations of T cells, macrophages, and smooth muscle cells in the human atherosclerotic plaque. Arteriosclerosis 1986;6:131-8.

15. Stoll G, Bendszus M. Inflammation and atherosclerosis: novel insights into plaque formation and destabilization. Stroke 2006;37:1923-32.

16. Tanaskovic S, Isenovic ER, Radak D. Inflammation as a marker for the prediction of internal carotid artery restenosis following eversion endarterectomy--evidence from clinical studies. Angiology 2011;62:535-42.

17. Johnston SC, Messina LM, Browner WS, et al. C-Reactive Protein Levels and Viable Chlamydia pneumoniae in Carotid Artery Atherosclerosis. Stroke 2001;32:2748-52.

18. Danesh J, Collins R, Peto R. Chronic infections and coronary heart disease: is there a link? Lancet 1997;350:430-6.

19. Libby P, Ridker PM, Hansson GK. Progress and challenges in translating the biology of atherosclerosis. Nature 2011;473:317-25.

20. Carr SC, Farb A, Pearce WH, et al. Activated inflammatory cells are associated with plaque rupture in carotid artery stenosis. Surgery 1997;122:757-63; discussion 763-4.

21. Grosse GM, Schulz-Schaeffer WJ, Teebken OE, et al. Monocyte Subsets and Related Chemokines in Carotid 
Artery Stenosis and Ischemic Stroke. Int J Mol Sci 2016;17:433.

22. Martinez E, Martorell J, Riambau V. Review of serum biomarkers in carotid atherosclerosis. J Vasc Surg 2020;71:329-41.

23. Köklü E, Yüksel İ, Arslan Ş, et al. Is Elevated Neutrophilto-Lymphocyte Ratio a Predictor of Stroke in Patients with Intermediate Carotid Artery Stenosis? J Stroke Cerebrovasc Dis 2016;25:578-84.

24. Gökhan S, Ozhasenekler A, Mansur Durgun H, et al. Neutrophil lymphocyte ratios in stroke subtypes and transient ischemic attack. Eur Rev Med Pharmacol Sci 2013;17:653-7.

25. Moro-García MA, Mayo JC, Sainz RM, et al. Influence of Inflammation in the Process of T Lymphocyte Differentiation: Proliferative, Metabolic, and Oxidative Changes. Front Immunol 2018;9:339.

26. Varım C, Varım P, Acar BA, et al. Usefulness of the platelet-to-lymphocyte ratio in predicting the severity of carotid artery stenosis in patients undergoing carotid angiography. Kaohsiung J Med Sci 2016;32:86-90.

27. Edsfeldt A, Nitulescu M, Grufman H, et al. Soluble urokinase plasminogen activator receptor is associated with inflammation in the vulnerable human atherosclerotic plaque. Stroke 2012;43:3305-12.

28. Rovina N, Akinosoglou K, Eugen-Olsen J, et al. Soluble urokinase plasminogen activator receptor (suPAR) as an early predictor of severe respiratory failure in patients with COVID-19 pneumonia. Crit Care 2020;24:187.

29. Torino C, Pizzini P, Cutrupi S, et al. Soluble Urokinase Plasminogen Activator Receptor (suPAR) and All-Cause and Cardiovascular Mortality in Diverse Hemodialysis Patients. Kidney Int Rep 2018;3:1100-9.

30. Debing E, Peeters E, Demanet C, et al. Markers of inflammation in patients with symptomatic and asymptomatic carotid artery stenosis: a case-control study. Vasc Endovascular Surg 2008;42:122-7.

31. Brinjikji W, Huston J 3rd, Rabinstein AA, et al. Contemporary carotid imaging: from degree of stenosis to plaque vulnerability. J Neurosurg 2016;124:27-42.

32. Puz P, Lasek-Bal A, Ziaja D, et al. Inflammatory markers in patients with internal carotid artery stenosis. Arch Med Sci 2013;9:254-60.

33. Mayer FJ, Binder CJ, Wagner OF, et al. Combined Effects of Inflammatory Status and Carotid Atherosclerosis. Stroke 2016;47:2952-8.

34. Wolak T. Osteopontin - A Multi-Modal Marker and Mediator in Atherosclerotic Vascular Disease.
Atherosclerosis 2014;236:327-37.

35. Carbone F, Rigamonti F, Burger F, et al. Serum levels of osteopontin predict major adverse cardiovascular events in patients with severe carotid artery stenosis. Int J Cardiol 2018;255:195-9.

36. DeMarco JK, Huston J 3rd. Imaging of high-risk carotid artery plaques: current status and future directions. Neurosurg Focus 2014;36:E1.

37. Pascoe MC, Crewther SG, Carey LM, et al. Inflammation and depression: why poststroke depression may be the norm and not the exception. Int J Stroke 2011;6:128-35.

38. Avgerinos ED, Kadoglou NP, Moulakakis KG, et al. Current role of biomarkers in carotid disease: a systematic review. Int J Stroke 2011;6:337-45.

39. Alserr AH, Elwan H, Antonopoulos CN, et al. Using serum s100- $\beta$ protein as a biomarker for comparing silent brain injury in carotid endarterectomy and carotid artery stenting. Int Angiol 2019;38:136-42.

40. Lin C, Tang X, Shi Z, et al. Serum tumor necrosis factor $\alpha$ levels are associated with new ischemic brain lesions after carotid artery stenting. J Vasc Surg 2018;68:771-8.

41. Zuniga MC, Tran TB, Baughman BD, et al. A Prospective Evaluation of Systemic Biomarkers and Cognitive Function Associated with Carotid Revascularization. Ann Surg 2016;264:659-65.

42. Vöö S, Kwee RM, Sluimer JC, et al. Imaging Intraplaque Inflammation in Carotid Atherosclerosis With 18F-Fluorocholine Positron Emission TomographyComputed Tomography: Prospective Study on Vulnerable Atheroma with Immunohistochemical Validation. Circ Cardiovasc Imaging 2016;9:e004467.

43. Rudd JH, Warburton EA, Fryer TD, et al. Imaging atherosclerotic plaque inflammation with $[18 \mathrm{~F}]-$ fluorodeoxyglucose positron emission tomography. Circulation 2002;105:2708-11.

44. Vesey AT, Jenkins WS, Irkle A, et al. (18)F-Fluoride and (18)F-Fluorodeoxyglucose Positron Emission Tomography After Transient Ischemic Attack or Minor Ischemic Stroke: Case-Control Study. Circ Cardiovasc Imaging 2017;10:e004976.

45. Hermus L, van Dam GM, Zeebregts CJ. Advanced carotid plaque imaging. Eur J Vasc Endovasc Surg 2010;39:125-33.

46. Goel S, Miller A, Agarwal C, et al. Imaging Modalities to Identity Inflammation in an Atherosclerotic Plaque. Radiol Res Pract 2015;2015:410967.

47. Kelly PJ, Camps-Renom P, Giannotti N, et al. Carotid Plaque Inflammation Imaged by (18)F-Fluorodeoxyglucose Positron Emission Tomography and Risk of Early 
Recurrent Stroke. Stroke 2019;50:1766-73.

48. Naghavi M, Libby P, Falk E, et al. From vulnerable plaque to vulnerable patient: a call for new definitions and risk assessment strategies: Part I. Circulation 2003;108:1664-72.

49. Cocker MS, Spence JD, Hammond R, et al. [18F]Fluorodeoxyglucose PET/CT imaging as a marker of carotid plaque inflammation: Comparison to immunohistology and relationship to acuity of events. Int J Cardiol 2018;271:378-86.

50. Mangge H, Almer G. Immune-Mediated Inflammation in Vulnerable Atherosclerotic Plaques. Molecules 2019;24:3072.

51. Tang T, Howarth SP, Miller SR, et al. Assessment of inflammatory burden contralateral to the symptomatic carotid stenosis using high-resolution ultrasmall, superparamagnetic iron oxide-enhanced MRI. Stroke 2006;37:2266-70.

52. Hermus L, Lefrandt JD, Tio RA, et al. Carotid plaque formation and serum biomarkers. Atherosclerosis 2010;213:21-9.

53. Camaré C, Pucelle M, Nègre-Salvayre A, et al. Angiogenesis in the atherosclerotic plaque. Redox Biol 2017;12:18-34.

54. Ding S, Zhang M, Zhao Y, et al. The Role of Carotid Plaque Vulnerability and Inflammation in the Pathogenesis of Acute Ischemic Stroke. Am J Med Sci 2008;336:27-31.

55. Andersson A, Hansebo G. Elderly peoples' experience of nursing care after a stroke: from a gender perspective. $\mathrm{J}$ Adv Nurs 2009;65:2038-45.

56. Chalela JA. Evaluating the carotid plaque: going beyond stenosis. Cerebrovasc Dis 2009;27 Suppl 1:19-24.

57. Tang TY, Howarth SP, Miller SR, et al. The ATHEROMA (Atorvastatin Therapy: Effects on Reduction of Macrophage Activity) Study. Evaluation using ultrasmall superparamagnetic iron oxide-enhanced magnetic resonance imaging in carotid disease. J Am Coll Cardiol 2009;53:2039-50.

58. Spence JD, Song H, Cheng G. Appropriate management of asymptomatic carotid stenosis. Stroke Vasc Neurol 2016;1:64-71.

59. World Stroke Organization. 1997. Available online: http://www.worldstrokecampaign.org/get-involved/wsoadvocacy-toolkit.html

60. Orrapin S, Rerkasem K. Carotid endarterectomy for symptomatic carotid stenosis. Cochrane Database Syst Rev 2017;6:CD001081.

61. Billington J, Deschamps I, Erck SC, et al. Developing Vaccines for SARS-CoV-2 and Future Epidemics and
Pandemics: Applying Lessons from Past Outbreaks. Health Secur 2020;18:241-9.

62. Andaluz N, Zuccarello M. Place of drug therapy in the treatment of carotid stenosis. CNS Drugs 2005;19:597-622.

63. Noiphithak R, Liengudom A. Recent Update on Carotid Endarterectomy versus Carotid Artery Stenting. Cerebrovasc Dis 2017;43:68-75.

64. Geovanini GR, Libby P. Atherosclerosis and inflammation: overview and updates. Clin Sci (Lond) 2018;132:1243-52.

65. Kirichenko TV, Sobenin IA, Nikolic D, et al. Anticytokine therapy for prevention of atherosclerosis. Phytomedicine 2016;23:1198-210.

66. Paraskevas KI, Cambria RP. Best Medical Treatment for Patients with Carotid Stenosis: Evidence-Based Medicine or Wishful Thinking? Angiology 2018;69:97-9.

67. Abbott AL. Bias in the use of randomized trials for carotid stenosis management. Gefasschirurgie 2015;20:252-7.

68. Constantinou J, Jayia P, Hamilton G. Best evidence for medical therapy for carotid artery stenosis. J Vasc Surg 2013;58:1129-39.

69. Liberale L, Bertolotto M, Carbone F, et al. Resistin exerts a beneficial role in atherosclerotic plaque inflammation by inhibiting neutrophil migration. Int J Cardiol 2018;272:13-9.

70. Chianese R, Coccurello R, Viggiano A, et al. Impact of Dietary Fats on Brain Functions. Curr Neuropharmacol 2018;16:1059-85.

71. Huo Y, Li J, Qin X, et al. Efficacy of folic acid therapy in primary prevention of stroke among adults with hypertension in China: the CSPPT randomized clinical trial. JAMA 2015;313:1325-35.

72. Husain S, Andrews NP, Mulcahy D, et al. Aspirin Improves Endothelial Dysfunction in Atherosclerosis. Circulation 1998;97:716-20.

73. Pascoe MC, Crewther SG, Carey LM, et al. Homocysteine as a potential biochemical marker for depression in elderly stroke survivors. Food Nutr Res 2012. doi: 10.3402/fnr. v56i0.14973.

74. Tsui PF, Lin CS, Ho LJ, et al. Spices and Atherosclerosis. Nutrients 2018;10:1724.

75. Otunola GA, Oloyede OB, Oladiji AT, et al. Selected spices and their combination modulate hypercholesterolemiainduced oxidative stress in experimental rats. Biol Res 2014;47:5.

76. Barter PJ, Caulfield M, Eriksson M, et al. Effects of torcetrapib in patients at high risk for coronary events. $\mathrm{N}$ Engl J Med 2007;357:2109-22.

77. Tardif JC, Kouz S, Waters DD, et al. Efficacy and Safety of Low-Dose Colchicine after Myocardial Infarction. N 
Engl J Med 2019;381:2497-505.

78. Nidorf SM, Eikelboom JW, Budgeon CA, et al. Lowdose colchicine for secondary prevention of cardiovascular disease. J Am Coll Cardiol 2013;61:404-10.

79. Ridker PM, Everett BM, Pradhan A, et al. Low-Dose Methotrexate for the Prevention of Atherosclerotic Events. N Engl J Med 2019;380:752-62.

80. Kim SC, Solomon DH, Rogers JR, et al. Cardiovascular Safety of Tocilizumab Versus Tumor Necrosis Factor Inhibitors in Patients With Rheumatoid Arthritis: A Multi-Database Cohort Study. Arthritis Rheumatol 2017;69:1154-64.

81. Ridker PM, Everett BM, Thuren T, et al. Antiinflammatory Therapy with Canakinumab for Atherosclerotic Disease. N Engl J Med 2017;377:1119-31.

82. Chen H, Luan X, Zhao K, et al. The association between neutrophil-to-lymphocyte ratio and post-stroke depression. Clin Chim Acta 2018;486:298-302.

83. Świtońska M, Słomka A, Korbal P, et al. Association of Neutrophil-to-Lymphocyte Ratio and Lymphocyte-toMonocyte Ratio with Treatment Modalities of Acute Ischaemic Stroke: A Pilot Study. Medicina (Kaunas) 2019;55:342.

84. Hyun S, Kwon S, Cho S, et al. Can the Neutrophil-toLymphocyte Ratio Appropriately Predict Carotid Artery

Cite this article as: Wijeratne T, Menon R, Sales C, Karimi L, Crewther S. Carotid artery stenosis and inflammatory biomarkers: the role of inflammation-induced immunological responses affecting the vascular systems. Ann Transl Med 2020;8(19):1276. doi: 10.21037/atm-20-4388
Stenosis in Patients with Ischemic Stroke?-A Retrospective Study. J Stroke Cerebrovasc Dis 2015;24:2646-51.

85. Zhang Y, Jiang L, Yang P, et al. Comparison of Lymphocyte Count, Neutrophil to Lymphocyte Ratio and Platelet to Lymphocyte Ratio in Predicting the Severity and the Clinical Outcomes of Acute Cerebral Infarction Patients. Clin Lab 2019. doi: 10.7754/Clin. Lab.2019.190102.

86. Huang G, Chen H, Wang Q, et al. High plateletto-lymphocyte ratio are associated with post-stroke depression. J Affect Disord 2019;246:105-11.

87. Kollikowski AM, Schuhmann MK, Nieswandt B, et al. Local Leukocyte Invasion during Hyperacute Human Ischemic Stroke. Ann Neurol 2020;87:466-79.

88. Farah R, Samra N. Mean platelets volume and neutrophil to lymphocyte ratio as predictors of stroke. J Clin Lab Anal 2018;32:e22189.

89. Jin P, Li X, Chen J, et al. Platelet-to-neutrophil ratio is a prognostic marker for 90-days outcome in acute ischemic stroke. J Clin Neurosci 2019;63:110-5.

90. Yilmaz E, Bayram Kacar A, Bozpolat A, et al. The relationship between hematological parameters and prognosis of children with acute ischemic stroke. Childs Nerv Syst 2018;34:655-61. 